\title{
Enhancing Performance Evaluation for Video Plagiarism Detection Using Local Feature through SVM and KNN algorithm
}

\author{
Ekta Thirani \\ Research Scholar, Faculty of Computer Engineering, Pacific Academy of Higher Education and Research University, \\ Udaipur. \\ Email: ektasarda16@gmail.com \\ Jayshree Jain \\ Department of Computer Engineering, Pacific Academic Higher Education and Research University, Udaipur, India \\ Email: drjayshreejain@gmail.com
}

\author{
Vaibhav Narawade \\ Department of Computer Engineering, Ramrao Adik Institute of Technology, Nerul. \\ Email: vnarawade@gmail.com
}

Received: 10 August 2021; Accepted: 06 September 2021; Published: 08 October 2021

\begin{abstract}
Nowadays in the digital world, there are lots of videos being uploaded to video, and social media sharing platforms are growing exponentially. About the Internet and multimedia technologies, illicitly copied content is a serious social problem. Since the internet is accessible to everyone, it is easy to download content and re-upload it. Copying videos from the internet can be considered plagiarism. In this paper, a method is proposed for feature extraction of video plagiarism detection. This framework is based on the local features to identify the videos frame by frame with the videos stored in the database. It becomes important to review the existing video plagiarism detection methods, compare them through appropriate performance metrics, list out their pros and cons and state the open challenges. First of all, it will pre-process the data with the help of SIFT and OCR Feature extraction. After that, the system applies the video retrieval and detection function using the two classifier algorithm the SVM, and the KNN. In the first stage, when the query is compared to all training data, KNN calculates the distances between the query and its neighbors and selects the K nearest neighbors. It is applied in the second stage to recognize the object using the SVM algorithm. Here we use the VSD dataset to predict the plagiarized videos. And the accuracy of these plagiarized videos after comparing them is $98 \%$.
\end{abstract}

Index Terms: Copyright protection, Euclidean distance and Video Processing, K-Nearest Neighbour (KNN), Support Vector Machine (SVM), video plagiarism detection, video copy detection.

\section{Introduction}

Video plagiarism detection is a process of detecting illegally copied videos by comparing them to the original content. Content management and copyright protection require effective and efficient plagiarism detection techniques. Over the last several decades, video plagiarism detection has been increasingly popular as a result of the growing awareness of video copyright.

Today, video plagiarism is a serious issue in the professional community, social media, and other content-sharing platforms. Yue Zhang [22] discusses that Infringement of film and video copyright concerns producers and distributors (e.g. movie, cable channels, streaming services). Video-sharing platforms have become increasingly popular (e.g. YouTube, Vimeo, etc) almost duplicate videos have been broadcast. Thus, in recent years, video plagiarism detection has gained a lot of interest due to the growing awareness for video copyright protection.

The ease with which videos can be downloaded and re-uploaded on other platforms makes it very important to detect duplicate videos to solve problems arising due to copyright violations. The statistics from Youtube show that approximately 400 hours of videos are downloaded every minute to Youtube, while about 30 million visitors per day observe approximately 5 billion hours of videos. The time people spent watching Youtube has doubled from the last 
year. Online video viewing - and the volume of online video content increases exponentially, as these statistics demonstrate. [1]

- Every week, $85 \%$ of people watch videos online, and $60 \%$ watch videos daily online. (HubSpot) (Spot)

- By 2020 , more than $90 \%$ of all consumer Internet traffic will make for online videos ( 85 percent in the US). (Switzerland)

- After Google, YouTube is the second most trafficked website. (Alexa) (Alexia)

Video sharing and social media platforms like Youtube, Netflix, Facebook, TVF are also widely distributed, as shown by statistics. So it becomes necessary to identify the illicit versions of original videos been uploaded on these platforms.

Various video plagiarism detection techniques exist broadly classified in content-based copy detection(CBCD) techniques and watermarking techniques. $\mathrm{CBCD}$ techniques find duplicate videos based on the content in the videos by analyzing frames of the videos and maintaining a database of the features extracted from original videos, later on comparing with query videos. Watermarking-based techniques embed metadata in the video that helps to detect whether the original video has been re-uploaded. Watermarking is not useful in the case of video transformations like blur, mirror, zoom in, zoom out due to post-processing and With compression, the possibility for disappearing appears.

Since there is no efficient and effective solution yet developed for video plagiarism. Therefore, our motivation is to develop a video detection solution for plagiarism.

Gitto George Thampi et al. and Robles [2,3] used Daubechies wavelet transform to obtain feature descriptors from video frames. The video is divided into several segments of fixed length (eg. 1 second) which have a fixed number of frames. The average of the successive features for each vector shall be obtained in each segment. A cluster of each video is formed and a cluster center is determined to facilitate similarity search, calculate the mean of representative vectors. Discrete wavelet transformations are used for a variety of reasons, including achieving different levels of detail or resolution, as well as being computationally appealing.

The SURF algorithm is a modern feature detector [5,4] that resembles the well-known SIFT features. Frames from the video are extracted and divided into 16 equal parts. The SURF points in each of the 16 cells are the descriptor. These vectors are stored in the database of each video. The descriptor sequentially compares each frame of the video with the query video frame. These methods are classified in the centre of the TRECVID copy detection submissions for the performance.

In this paper [13], first, to represent semantic information, the visual features (locals and global features) are extracted. These features are extracted at various levels. The global features are used to quickly filter out the candidate videos similar to the query video.

Chaitra Ravi [16] superior efficiency can be obtained in terms of time and energy that are implemented suing machine learning algorithms. Due to this high-speed capability, the FAST (Features from Accelerated Segment Test) corner detector is very useful for real-time video processing applications.

The fast matching sequence technique uses the Edit-distance-based approach. An Edit distance in the context of video matching is used for matching two sequences. Edit distance between two strings $A=[a 1, a 2, \ldots, a m]$ and $B=[B 1$, $\mathrm{B} 2, \ldots, \mathrm{Bn}]$, over an alphabet $\sum$ is the lowest cost of executing a series of operations that converts A into B [6] . A matching approach for assessing similarity is implemented in called as the Longest Common Subsequence [7,8]a and Adaptive vocabulary tree is a better approach for the vocabulary tree.

The short-length Sequence technique uses temporal features for detecting video copy detection. The black border removal is introduced in this paper that are necessary to improve the system performance [9] .

In video printing using Hadoop, Calculates the distance between the video hash query and the database hash. The video is copied if the minimum distance is less than the threshold value [10]. Different classifiers are applied to local features. Local features play a crucial role in the recognition of licence plates from video [14]. Two-step strategy devised by Kharat and Chougule [15] to identify suspicious frames in video sequences. As a result of this work, SIFT descriptors were used to describe SIFT. This method was used to extract feature vectors or vectors of interest from the data. The keypoints from the frames were compared to the frames themselves. Assigning keypoints with other frames in order to reach a conclusion .finally, a random sample consensus approach was used in the research. When detecting duplicate frames, this method is used. 'In this case,' says the as part of the two-step approach, literature was reviewed and analysed. Uncompressed videos were tested, as were compressed videos. Rate of compressed data can be changed. Experimentation with a resultant indicated that the established two-step technique was effective and efficient. Detection of manipulated products is now more accurate. Compared to the preceding techniques, the new frames are much smaller. Bhatt, P.P $[18,19]$ define that OCR model can be implement in detecting and identifying a binding text for text areas in the image and the characters. KNN gives high accuracy as compared to linear regression and Naive Bayes but its time complexity is also high as compared to other machine learning algorithms.

It proposed that a local feature of object detection in the image is used from the literature review. A new classifier type is also built on this paper to hybridize KNN and SVM. Local video frame descriptors are integrated into one vector, reducing the vector dimension. The index able video is encoded using a temporal indexing system. The query 
framework descriptors are not encoded. The final ranking of database videos relevant to the query is determined by a weighted temporal Hough transformation. A weighted temporal Hough transform provides the final rating of database videos relevant to the query.

The rest of this paper is describing in this way. Stage II describes Feature Extraction. Stage III define and describe the Classification methods. Stage IV describes proposed method. Stage V analysis and result discuss. Finally, VI conclusion and future research.

\section{Feature Extraction}

First of all, we convert the video into a framework for the copy detection model. Data must be converted into a classifier format, which is called feature extraction. In this paper we consist three types of features extraction method to achieve local feature detection for video plagiarism. The first SIFT function is used to describe the local characteristics of this image. OCR is the second factor. After this we found the shape and brightness of the image like image resizing, blurring and image has be calculated to local features in an image.

I. Feature extraction using SIFT: - (Scale-Invariant Feature Transform) The scale-invariant feature transform (SIFT) is a feature detection technique, SIFT, is used in computer vision to recognise and characterise local features in images. In the below fig. 1 after the pre-processing of the videos is done, the SIFT points are calculated for each video frame by frame. In fig. 2 apply SIFT algorithm to detect the descriptors and the key points of each frame are recorded and all descriptors and key points are stored in one pickle file. A Python object structure may be serialised and de-serialized with Pickle. It is possible to save python objects onto a hard drive by "pickling" them. In general, it's important that to convert the video into a framework for the copy detection model. Feature extraction is called the process of converting the data in a classifier format required.

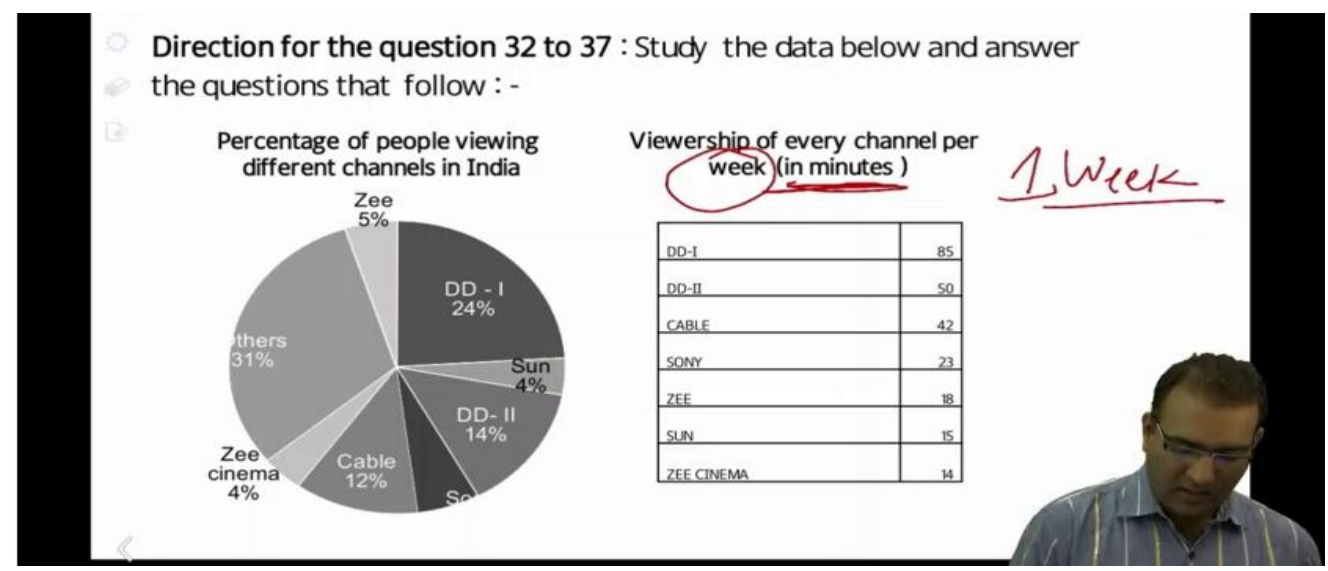

Fig.1. Capturing the video image

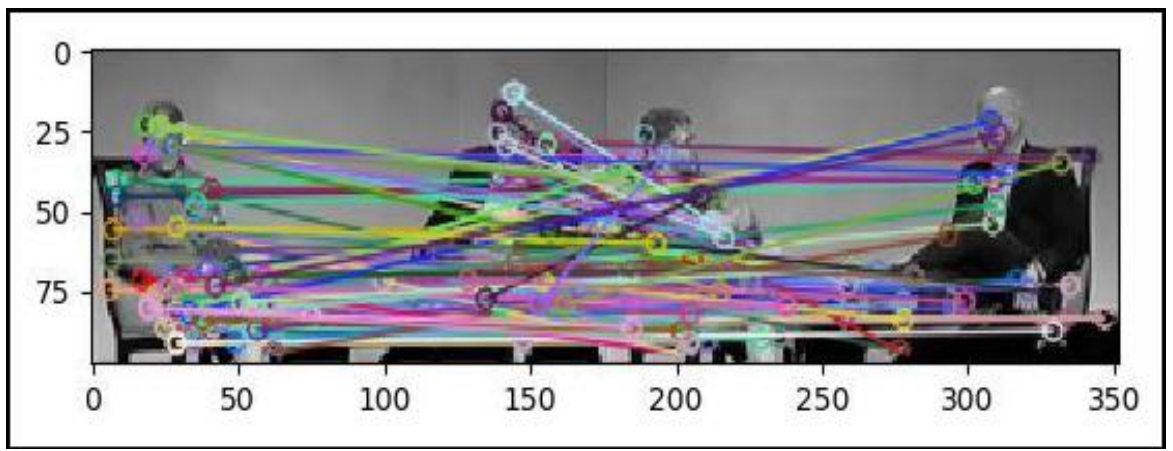

Fig.2. Feature Matching SIFT algorithm

II.OCR:(Optical Character Recognition): after feature descriptor we will apply the remaining part in OCR to detect the text image in the video. Tesseract is an open-source OCR engine it takes images that attempt to acknowledge the text. The result is a text string, and the degree to which an image is similar to a human-readable text is measured by its correctness [17]. The OCR is used to recognize printed documents in papers, handwritten characters, or physical text messages such as license plates, street signs, and street numbers, document account holders, legal forms, ID cards, and so on. The detected field is given by OCR to extract the text from detected image and is then stored in a CSV file. In below fig. 3 shows the text to be detected in video frame. 


\begin{tabular}{|c|c|c|}
\hline 0 & Direction for the question 32 to 37 : Study th... & 1.mp4 \\
\hline 1 & Direction for the question 32 to 37 : Study th... & 1. $\mathrm{mp} 4$ \\
\hline 2 & Direction for the question 32 to 37 : Study th... & 1.mp4 \\
\hline 3 & Direction for the question 32 to 37 : Study th... & 1.mp4 \\
\hline 4 & Direction for the question 32 to 37 : Study th... & 1. $\mathrm{mp} 4$ \\
\hline$\cdots$ & $\cdots$ & $\cdots$ \\
\hline 1465 & 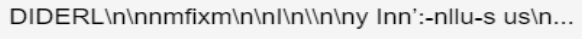 & 9.mp4 \\
\hline 1466 & 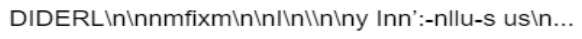 & 9.mp4 \\
\hline 1467 & 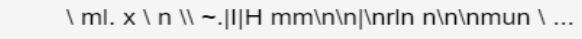 & 9.mp4 \\
\hline 1468 & $\ln \backslash n$ & 9.mp4 \\
\hline 1469 & $\ln \backslash n$ & 9.mp4 \\
\hline
\end{tabular}

Fig. 3. Text detected through Tesseract OCR

So here, all the features are detected using SIFT and OCR to achieve my research objective in video that are converted into image found to generate some plagiarized video in an image by using feature extraction techniques.

\section{Feature Matching}

After feature extraction we can apply the feature matching technique based on classification algorithm such as KNN and SVM to compare training and testing data image in a frame. To detect the video are plagiarised or not based on that first of all we define KNN algorithm after that we compare with SVM classifiers.

\section{K-Nearest Neighbour: -}

Dasarathy [20] was introduced first machine learning in 1919 i.e K Nearest Neighbour. The nearest neighbour algorithm for data mining and the statistic pattern recognition is a fast, strong, and easy-to-understand supervised machine learning used in several applications. KNN algorithm to solve problems of both classification and regression. [21] Hug, describe that it is one way to classify objects using the nearest training images in the extraction feature. In $\mathrm{kNN}$ where $\mathrm{k}$ helps us to identify clusters for an object, the positive integer of its neighbors has been classified. If $\mathrm{k}=1$ or $\mathrm{k}=2$, the object can be noisy and cause outliers in the model to have effects. It's easy to predict a data class training data from stored with its label for all classification and regression algorithms. To predict a test example, calculate first your distance to the example training. Keep the $\mathrm{k}$ examples closest to each other, where $\mathrm{k}$ is always bigger than one integral. $\mathrm{K}=5$, for instance. In equation no. 2 the distance feature used is the Euclidean distance between two ends when each example is a vector of fixed length in real number.

$$
\mathrm{ED}(\mathrm{P}, \mathrm{Q})=\left((\mathrm{P} 1-\mathrm{Q} 1)^{2}\left((\mathrm{P} 2-\mathrm{Q} 2)^{2}+\ldots+\left(\mathrm{P}_{\mathrm{n}}-\mathrm{Q}_{\mathrm{n}}\right)^{2}\right)^{2}\right.
$$

Where $\mathrm{P}$ and $\mathrm{Q}$ are two category points with $\mathrm{n}$ characteristics. And there is another method to calculate Euclidean Distance which is known as Manhattan distance.

\section{SVM (Support vector machine): -}

The Support vector machine is an algorithm based on supervised learning and used for classification and regression, the problem belonging to generalized linear classification. SVM is mainly a method used in the identification of patterns and objects. If anyone can be found in N-dimensional space, the primary purpose of the support vector is to identify some functions that classify data points separately. For facial detection, image classification, etc., users can use the SVM algorithm. The decide surface is called the hyperplane. The maximum and minimal separation between the hyperplane is based on positive and negative support vectors of the hyperplane.

SVM consists of two types: Linear SVM can, using a single straightened line, use data for linearly separable information and its classifiers to two classes, then be called Linear SVM classifier. SVM consists of two types of data: Non-linear SVM: uses the separated data and cannot classify the direct line, is called non-linear, and classification is called non-linear SVM classification.

For the proposed work, after retrieving the function for the feature extraction with the training sample to maximize the margin between the data points and hyperplane. The loss function that supports margin optimization is loss is defined in equation no. 2

$$
\mathrm{C}(\mathrm{x}, \mathrm{y}, \mathrm{f}(\mathrm{x}))=(1-\mathrm{y} * \mathrm{f}(\mathrm{x}))
$$

The left-hand function may be represented as a right-hand function. If the present value and the actual value are the same sign, the cost is 0 . Otherwise, the loss value is computed. We are also adding a parameter control cost feature. The 
goal is to balance margin with maximizing losses. After adding the controlled parameter, the cost functions are presented below in equation no. 3 and 4.

With the loss function now, we're picking up partial derivatives to find the gradients.

$$
\begin{gathered}
\operatorname{minw}^{\gamma}\|w\| 2+\sum_{i=1}^{n}(1-y i(x i, w)) \\
\partial / \partial_{\mathrm{k}}\left(1-\mathrm{y}_{\mathrm{i}}(\mathrm{xi}, \mathrm{w})\right)_{+}= \begin{cases}0 & \text { if } \mathrm{y}_{\mathrm{i}}\left(\mathrm{x}_{\mathrm{i}}, \mathrm{w}\right)>=1 \\
-\mathrm{y}_{\mathrm{i}} \mathrm{x}_{\mathrm{ik}} \text { else } & \end{cases}
\end{gathered}
$$

If no errors occur, our pattern predicts correctly and the class of our data point. Our model predicts correctly and of our data point. The gradient of the adjustment parameter must only be updated by using equation no.5.

$$
\mathrm{W}=\mathrm{w}-\alpha \cdot(2 £ \mathrm{w})
$$

If the error occurs, our model makes an error in the class prediction of our data point, we include the loss with the regulation parameter for updating the gradient in equation no. 6 .

$$
\mathrm{W}=\mathrm{w}+\alpha \cdot\left(\mathrm{y}_{\mathrm{i}} \cdot \mathrm{x}_{\mathrm{i}}-2 £ \mathrm{w}\right)
$$

After this feature Extraction matching part, we found that most of the features such as text, images, colour brightness can achieve the objective based on that we can apply classification algorithm for further process.

\section{Proposed Method}

In this proposed methodology, we can take a different input videos in our database after that we apply feature extraction and feature matching algorithm to find out the plagiarized images in our video then we can apply some query video for the classification system may enhance the video copy detection performance. The SVM is a linear classification for high-dimensional feature space recognition and thus suited for classification for images and objects. KNN algorithm applies to find out the nearest values for the images. SVM-KNN classifier applies the image are given as input into the proposed method for query video. The detailed explanation is described below in fig.no.4.

\section{Algorithm:}

i) The input video was acquired and the video is pre-processed for extraction of feature using SIFTS, OCR, and image hash.

ii) The vectorial component of the given image is being constructed.

iii) Distance functions like the Euclidean Distances indicate the closest neighbors in the K-Nearest Neighbor.

iv) The query database video will be labelled and dropped when neighbors $\mathrm{K}$ all have the same tag. otherwise, calculate pairs of distances between their neighbors $\mathrm{K}$ and construct the distance matrix. And convert it into the kernel matrix and use SVM.

v). To label the query, use the resulting SVM classification.

In the proposed method, fig. 4 defined that the given video after taking the input video in MPEG-4 format preprocessing is done. Following are the steps for pre-processing.

1.Reducing frames per second(fps). (2fps).

2.Converting to grayscale.

3.Decreasing resolution(480x360).

After pre-processing to extract the image feature, for SIFT, a method of detecting the OCR and images is applied. It is possible to determine invariants after the edge has been removed. To find the nearest neighbours to a particular image, KNN is applied to all training images. If the algorithm terminates when a label is found, the SVM is used in the other case. The video is considered a plagiarized video and is flagged accordingly to recognize the object. 


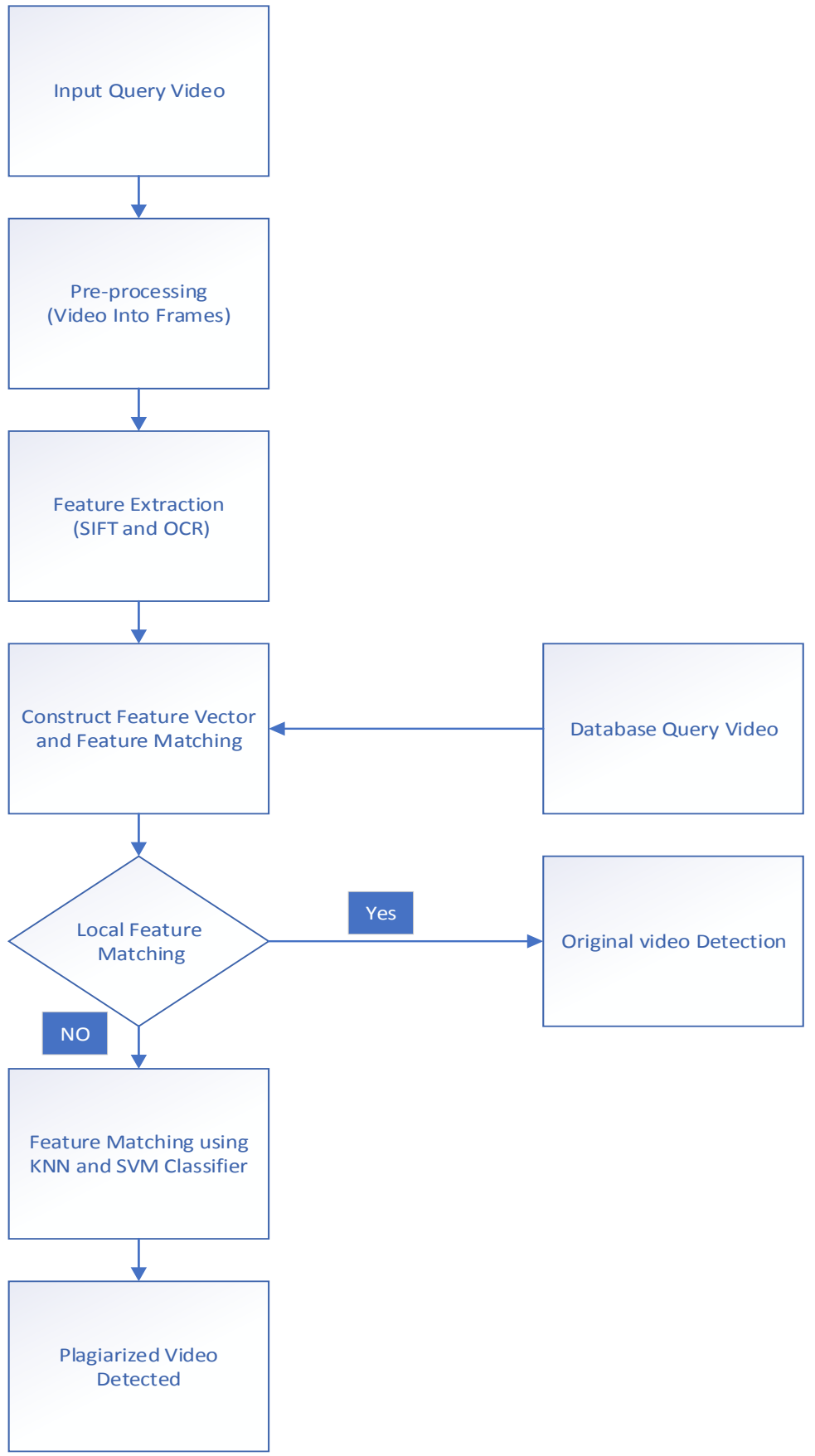

Fig.4. Proposed Methodology

\section{Experimental Result \& Performance Evaluation}

The method has proposed local identification of the image functions using SIFT, OCR, Imagehash, and after the image has been trained in SVM and KNN classification. VCDB dataset can be used to segregate videos into the Sports, Cooking, Gaming, and Film Trailer databases with the 100 videos. It takes approximately 10 seconds for a video to preprocess and extract features that include a reduction in fps, resizing, gray scaling, store keypads, and descriptors in the package. We, therefore, need time to process about half the length of the video.

Each database contains 25 videos, each rotated at a vertical axis with a 5-degree angle interval, with 250 different black background images. There also are approximately 4000 images for each object, totalling up to 40,000 images in the database as a whole. All unique videos are pre-processed and features are extracted at a certain frame rate from frames of the videos. The OCR detects local characteristics and the image hash is detected for all image characteristics. These are the example of VCBD dataset videos. 


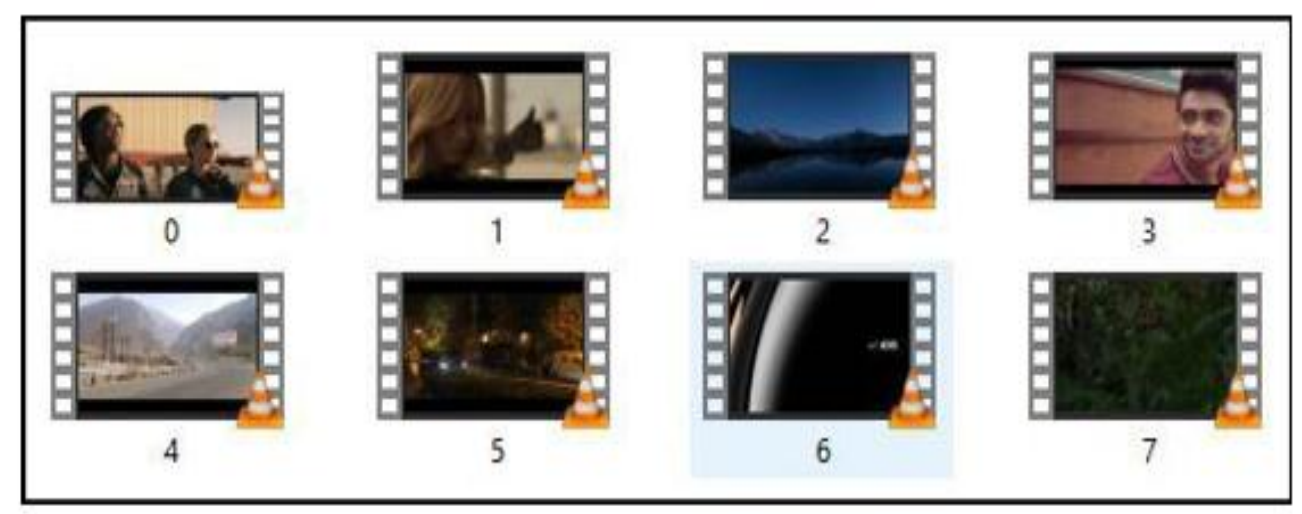

Fig.5. Dataset VCDB

In the next step, the KNN classification algorithm determines $\mathrm{x} 0$ 's $\mathrm{K}$ closest neighbours. Where $\mathrm{x} 0$ is a training image, testing the image vector. Without prior knowledge, Euclidian distance is a metric distance by the KNN classifier. As soon as the KNN is completed, the question is labelled, and the programme is terminated automatically. To assess the Distance matrix, we shall use a kernel matrix. Otherwise, a label of the object shall be applied to the SVM classifier for a kernel matrix. For various training sets and VCDB-1000 test images, that was used in proposed method as shown in table 1 below.

Table 1. Based on the following factors, the accuracy of each categorization technique may be determined.

\begin{tabular}{|l|l|l|l|l|l|}
\hline Transformation & Frames & Precision & Recall & F-Score & Accuracy \\
\hline Shape & 91 & 0.850049 & 0.851469 & 0.850749 & $79.99 \%$ \\
\hline Brightness & 91 & 0.297954 & 0.892268 & 0.799635 & $89.00 \%$ \\
\hline Image contrast & 91 & & & & $90 \%$ \\
\hline Bars & 92 & 0.955189 & 0.849307 & 0.752233 & \\
\hline Hue & 91 & 0.855346 & 0.875642 & 0.33432 & $87 \%$ \\
\hline Extra1 & 95 & 0.937311 & 0.944615 & 0.835198 & $83 \%$ \\
\hline Extra 2 & 93 & 0.941860 & 0.843660 & 0.939086 & $94 \%$ \\
\hline Mixed & 109 & 0.875264 & 0.790272 & 0.942741 & $93 \%$ \\
\hline
\end{tabular}

Proposed method performance compared to the K-NN Classifier. If all items are trained and tested Pre-processing Settings Computing Performing Invariant Construct the Vector feature that plagiarized video.

The performances are evaluating on the basis of Recall, Precision, F- score and Accuracy. During the performance evaluation, some open-source machine learning algorithms were implemented in this research. Here we give the accuracy of all algorithms on 100 videos. Precession, recall, F-Score, and Accuracy are the four attributes that emerged from the results. Similarly, recall is the proportion of relevant examples that are found, the number of relevant occurrences that are detected is called precision (also known as positive predictive value). As a result, precision and recall are both dependent on knowledge of relevance and a method for assessing it. Precision and recall are used to calculate F-score. To properly analyse the results, Graphs should be used to compare them. 


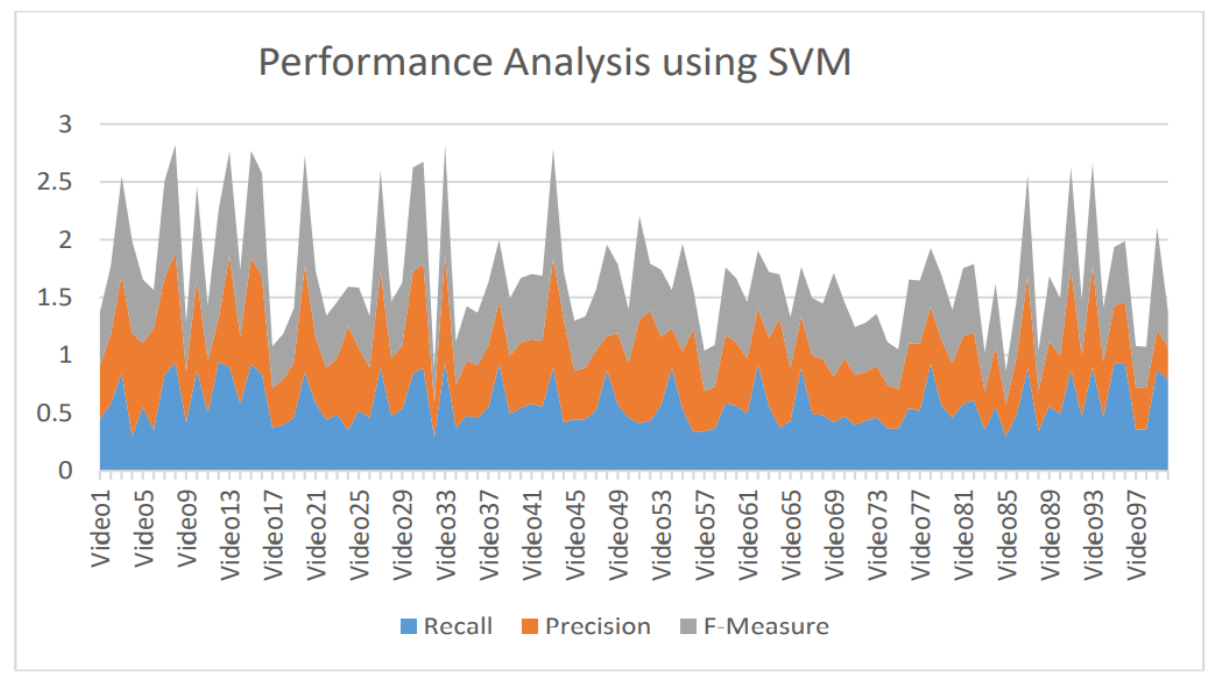

Fig. 6. Performance Analysis using SVM

In fig.6. The SVM's accuracy is shown. SVM with four features has an accuracy of roughly $80 \%$ for different dataset sizes, as can be seen in the graph below (right). As a result of normalising the data set, this value increases. With the addition of another feature, keyword-based features, the accuracy of each dataset increases. Using grid search, we finally achieve an accuracy of 94 percent for each data set. SVM with four features has an accuracy of roughly $96 \%$ for different dataset sizes, as can be seen in the graph below (right). As a result of normalising the data set, this value increases.

Using the number of true positives divided by both the number of true positives and the false negatives, the recall can be calculated. Using the number of true positives divided by the number of false positives, precision is calculated. F1 score is calculated using precision and recall. It provides the system with overall performance. These are the formula we can use to find the accuracy precision and Recall and F1- Score apply using the SVM and KNN algorithm for classification.

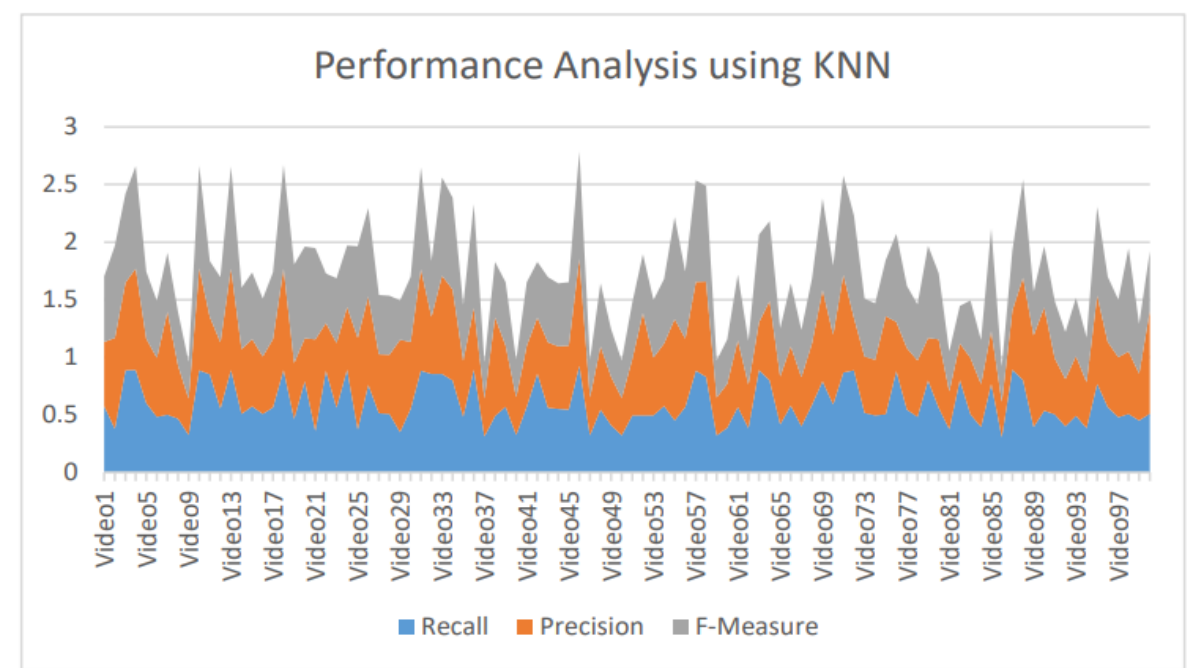

Fig.7. Performance Analysis using k-nearest neighbour.

In the initial KNN (four features), we observe that the precision is less than that of the updated KNN, which has four features but normalised data. With the previous version of KNN, an image-based feature was added that increased accuracy even more. There are several variants of KNN, each with a high recall for different size data sets. This is a good thing since it means that we are able to boost recall from the prior edition to about 80 percent, which is acceptable. Also, the F1 score value will be computed. SVM's accuracy and recall are greater than VCSD's Knn when comparing these performance parameters. We can conclude that based on precision, SVM performs better than KNN.

The benefits of using the SVN has better the KNN algorithm in video plagiarism detection has good accuracy. The average accuracy in video plagiarism detection is $98 \%$. The algorithm works well for various transformations in the videos. 


\section{Conclusion}

In this paper, we provide a local video copy detection method based on attribute such as SIFT, OCR, Imagehash, and brightness for local feature. KNN-SVM is the classifier used by the vector to identify an object. The KNN classifier is first applied when the nearest object of the trained characteristics is not identified; the object is identified by SVM. In the proposed method, the local feature detection in the video gives more accurate. The table results are found based on their classification algorithm to implement the performance metrics. It is evident that it can produce better results when combining SVM and KNN with local functionality. A polynomial function is used for the suggested method as a kernel function. More techniques need to be developed which will be efficient, effective as well as the computational power required is less.

The time required for computation also needs to be minimized. In future research, one may consider applying more complex and different technique for achieving great accuracy in plagiarism detection in videos. The global feature detection and extraction techniques can be used together with Hadoop so that the database can be easily managed and more techniques need to be discovered for better accuracy and results.

\section{References}

[1] Video Marketing Statistics https://biteable.com/blog/tips/video-marketing-statisti cs.

[2] Gitto George Thampi, D. Abraham Chandy, "Content-based video copy detection using discrete wavelet transform", in IEEE Proc.of Conf. on Information and Communication Technologies, 2013.

[3] Robles, Oscar D., et al. "Towards a content-based video retrieval system using wavelet-based signatures," 7th IASTED International Conference on Computer Graphics and Imaging-CGIM, 2004.

[4] Gerhard Roth, Robert Laganiere, Patrick Lambert, Ilias Lakhmiri, and Tarik Janati “A Simple but EffectiveApproach to Video Copy Detection" in Canadian Conference Computer and Robot Vision, 2010.

[5] H. Bay, A. Ess, T. Tuytelaars, and L. J. V. Gool. Speeded-up robust features (surf). Computer Vision and Image Understanding, 110(3):346-359, 2008

[6] Yeh, M.-C., \& Cheng, K.-T. (2009). Video copy detection by fast sequence matching. Proceeding of the ACM International Conference on Image and Video Retrieval, 2009

[7] Y. Kim, and T. -S. Chua. Retrieval of news video using video sequence matching. In Proceedings of the International Multimedia Modelling Conference (MMM'05), pages 68-75, 2005.

[8] T. Yeh, J. Lee, and T. Darrell. Adaptive vocabulary forests for dynamic indexing and category learning. In Proceedings of the IEEE International Conference on Computer Vision (ICCV’07), pages 1-8, 2007.

[9] Janya Sainui, Ladda Preechaveerakul, Lekha Chaisorn, "An image based video copy detection using ordinal bitmap signature", in IEEE Proc.of int. conf. on Information, Communications and Signal Processing, pages 1-5, 2011

[10] Li, J., Wu, Q., Lian, X., \& Sun, J., "Real-time video copy detection based on Hadoop" Sixth International Conference on Information Science and Technology (ICIST), 2016.

[11] M. Douze, H.Jegou,C.Schmid, and P. Perez, "Compact video description for copy detection with precise temporal alignment", ECCV, 2010

[12] D. G. Lowe, "Distinctive image features from scale invariant keypoints", in Int. Journal on Comput. Vision, pp. 91-110, 2004.

[13] Xiaoguang Gu, Dongming Zhang, Yongdong Zhang, Jintao Li, Lei Zhang, "A video copy detection algorithm combining local features robustness and global feature's speed", in IEEE Proc. of Int. Conf.

[14] X.He, H.Zhang, W.Jia, Q.Wu and T.Hintz, Combining Global and features for detection of License plates in a video, Proceedings of Image and Vision Computing, 2007, pp. 288 - 293.

[15] J. Kharat and S. Chougule, "A passive blind forgery detection technique to identify frame duplication attack", Multimedia

[16] Chaitra Ravi, Rajaram M Gowda. "Development of Image Stitching Using Feature Detection and Feature Matching Techniques", 2020 IEEE International Conference for Innovation in Technology (INOCON), 2020.

[17] Mirza, A., Zeshan, O., Atif, M., \& Siddiqi, I. (2020). Detection and recognition of cursive text from video frames. EURASIP Journal on Image and Video Processing, 2020(1), 1-19.

[18] Bhatt, P. P., \& Patel, I. (2018). Optical Character Recognition Using Deep Learning-A Technical Review. National Journal of System and Information Technology, 11(1), 55.

[19] Bijalwan, V., Kumar, V., Kumari, P., \& Pascual, J. (2014). KNN based machine learning approach for text and document mining. International Journal of Database Theory and Application, 7(1), 61-70.

[20] Dasarathy, B. V. (1991). Nearest neighbor (NN) norms: NN pattern classification techniques. IEEE Computer Society Tutorial.

[21] Huq, M. R., Ali, A., \& Rahman, A. (2017). Sentiment analysis on Twitter data using KNN and SVM. International Journal of Advanced Computer Science and Applications, 8(6), 19-25.

[22] Zhang, Y., \& Zhang, X. (2016, December). Effective real-scenario video copy detection. In 2016 23rd International Conference on Pattern Recognition (ICPR) (pp. 3951-3956). IEEE. 


\section{Author's Profiles}

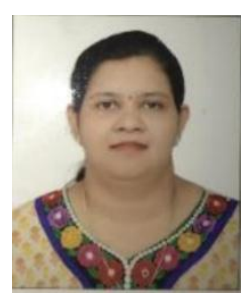

Ms. Ekta Sarda is currently working as an assistant professor in Computer engineering department, RAIT. She is having 15 years of teaching experience. She has completed her ME in computer science and engineering from Rajasthan Technical university kota and pursuing PHD in computer engineering from PAHAR University. Her area of specialization is Pattern Recognition. She has published paper in 6 journals and 3 international conference and 1 book chapter. She has also published one book in Computer Network.

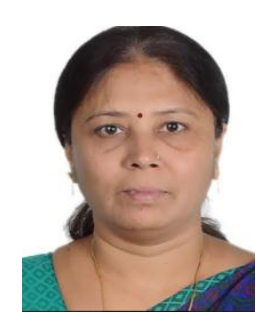

Dr. Jayshree Jain is a professor in Pacific University. After graduating in IT, she did MTech, MBA and PhD. Her research area in Doctorate was \& quot;Potential of e-Learning in Indian Education System \& quot;. She is the life member of CSI, IIIE, ISTD and many other professional bodies. He has been the member of organizing committee for various national and international seminars / workshops. Dr. Jain has also completed SCJP Certification.

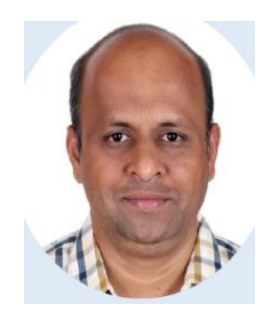

Dr. Vaibhav Narawade is a Professor in the Ramrao Adik Institute of Technology at Nerul affiliated to University of Mumbai where he has been a faculty member since 2019. Professor Narawade area of expertise includes Wireless Sensor Network, Image Processing, and Data Science. He has having and Administrative He has served University of Mumbai as Member 2010) and currently also served as Member of Management Council and Member of Senate, University of Mumbai (2017 to 2022), In Academics he is a n Computer Science and Engineering, Information Technology fraternity, and Faculty Narawade has author / coauthored in various International conference / journal publications and book chapter. He is a fellow of ISTE and ACM.

How to cite this paper: Ekta Thirani, Jayshree Jain, Vaibhav Narawade, " Enhancing Performance Evaluation for Video Plagiarism Detection Using Local Feature through SVM and KNN algorithm", International Journal of Image, Graphics and Signal Processing(IJIGSP), Vol.13, No.5, pp. 41-50, 2021.DOI: 10.5815/ijigsp.2021.05.04 